\title{
Leçons tirées de l'accident d'un accélérateur linéaire de l'hôpital de Saragosse le 5 décembre 1990 : organisation de la physique médicale et de la radioprotection en Espagne
}

\author{
L. ARRANZ ${ }^{1}$
}

(Manuscrit reçu 16 juin 2009, accepté le 15 octobre 2009)

RÉSUMÉ L'accident de l'hôpital de Saragosse, en 1990 a considérablement modifié la réglementation espagnole en matière de radioprotection des patients. Ces modifications se sont faites en même temps que la transposition de nouvelles directives européennes faisant suite à la directive européenne de 1991. Nous décrivons ici l'historique des modifications réglementaires espagnoles que cet accident a accéléré.

ABSTRACT Learned lessons from the crash of a linear accelerator in Zaragoza Hospital, December 5, 1990 .

The accident of the hospital of Zaragoza, in 1990 considerably modified the Spanish regulation as regards protection against radiation of the patients. These modifications were done at the same time as the transposition of new European directives making following the 1991's ICRP recommendations. We describe the history of the Spanish lawful modifications here that this accident accelerated.

Keywords: radiological accident / regulation / Spain

\section{Introduction}

L'organisation de la radioprotection dans le secteur médical en Espagne a connu, d'un point de vue historique, deux étapes bien définies.

La première, dans les années 80 , avec :

- la création du Conseil de sécurité nucléaire (CSN), unique autorité de contrôle en la matière, indépendant du gouvernement (Loi, 1980),

- l'adéquation de la législation espagnole aux recommandations de la CIPR de 1977 (ICRP, 1977) pour la réglementation de la protection sanitaire contre les radiations ionisantes (Décret royal, 1982),

\footnotetext{
1 Service de radiophysique et radioprotection, Hôpital Universitaire « Ramón y Cajal », E-28034 Madrid, Espagne.
} 
- la nouvelle loi générale de la santé, qui oblige à établir les requêtes minimales pour l'approbation et l'homologation des installations en relation à la sécurité, et son efficacité (Loi, 1986),

- la transposition de la directive 84/466/Euratom sur la radioprotection du patient (CCE, 1984), du fait de l'entrée de l'Espagne dans la CEE en 1986 (Décret royal, 1990),

- la création et la dotation des services de radioprotection dans les grands hôpitaux publics de l'institut national de la santé du ministère de la santé (MSC, 1990).

Lors de cette première étape il a été créé la fonction de «personne compétente en radioprotection » $(\mathrm{PCR})$ appelée en Espagne « chef de service de radioprotection » (Décret royal, 1982), en accord avec la directive 84/836/Euratom (CCE, 1984) et la fonction d'«expert qualifié en radiophysique» (qui deviendra ensuite « spécialiste en radiophysique hospitalière », 1990), en accord avec cette directive Euratom. Le CSN avait déjà proposé aux autorités sanitaires de créer des services de radioprotection dans les établissements de santé disposants d'installations de radiothérapie, de médecine nucléaire ou de radiodiagnostic, avec des missions bien définies en ce qui concerne la radioprotection des travailleurs et du public. Beaucoup d'hôpitaux disposaient déjà de physiciens médicaux qui travaillaient dans les services de radiothérapie, de médecine nucléaire ou de radiodiagnostic, mais comme leur rôle n'était pas réglementé il n'existait pas de contrôle de leur formation ni de planification, au niveau national, de leurs missions.

La deuxième étape, dans les années 90, a été marquée par divers faits :

- l'accident de l'accélérateur linéaire de l'hôpital clinique de Saragosse, qui mit en évidence une série de dysfonctionnements (CSN, 1991; SEFM, 1991),

- la publication des nouvelles recommandations de la CIPR (ICRP, 1991), des normes de base de l'IAEA (1994) et des directives européennes reprenant ces recommandations, dans les années 1996 et 1997,

- la création réglementaire de « spécialiste en radiophysique hospitalière » dans le système national des spécialités de la santé (Décret royal, 1997a).

Tout cela eu comme conséquence, la réglementation des critères de qualité dans les installations de radiothérapie, de médecine nucléaire et de radiodiagnostic, par le ministère de la Santé, avec l'accord favorable du CSN entre 1997 et 1999 (Décret royal, 1997b, 1998, 1999). 


\section{L’accident de l'accélérateur linéaire de Saragosse}

Le 5 décembre de 1990, les personnes en charge de traiter des patients avec un accélérateur linéaire CGR MeV modèle Sagittaire (25 MV en régime de photons et jusqu'à $40 \mathrm{MeV}$ en régime d'électrons) de l'hôpital clinique de Saragosse, observèrent que l'équipement ne fonctionnait pas, et n'émettait pas d'électrons. L'origine était un court-circuit dans le circuit de contrôle de l'intensité du courant (Arranz, 2008).

- Les opérateurs, conformément au processus interne, auraient du prévenir les physiciens, le responsable médicale de l'installation et le responsable du service technique de l'hôpital, et enregistrer l'incident dans le «journal d'opération ». Au lieu d'informer les responsables de l'installation, ils se limitèrent à communiquer oralement cet incident au technicien d'entretien de Général Electric-CGR, qui, était alors en train de réparer une unité de télé cobaltothérapie dans une salle annexe.

- Le technicien d'entretien n'informa pas le responsable du service technique de l'hôpital comme prévu dans le contrat officiel d'entretien.

- Après une première réparation incorrecte ce même jour (il agit sur l'énergie des électrons au lieu de réparer le court-circuit produit), le technicien de GE-CGR élimina inconsciemment le contrôle d'énergie que l'opérateur de l'accélérateur doit effectuer pour chaque traitement dans le pupitre de contrôle : l'indication analogique de l'énergie des électrons montraient toujours $36 \mathrm{MeV}$, bien que les touches de sélection de l'énergie correspondaient à 7, 10 ou $13 \mathrm{MeV}$.

- Le technicien communiqua aux opérateurs de l'accélérateur que l'indicateur analogique ne fonctionnait pas et qu'il avait commandé un nouvel indicateur pour son remplacement.

Le 10 décembre, les traitements reprennent. Mais pour les traitements avec électrons les doses absorbées, à la profondeur de référence que fournissait l'accélérateur pour chaque faisceau de radiation, étaient, logiquement, supérieures à la dose prescrite. Dans les irradiations avec photons, il ne se produit pas de déviations significatives du point de vue de la sécurité (SEFM, 1991).

Au bout de 10 jours (les médecins observèrent des troubles et intolérance chez quelques patients), les opérateurs communiquèrent aux physiciens le blocage de la signalisation analogique. Les physiciens ont réalisé immédiatement un contrôle dosimétrique et vérifièrent que les électrons émis avaient toujours la même énergie de $36 \mathrm{MeV}$. Les traitements ont alors été suspendus.

Le rapport de «l'Institute of Medecine » (Kohn et al., 1999) « To error is human» indique que les systèmes d'organisation du travail, dans le milieu 
sanitaire, sont habituellement complexes et quand ils échouent, c'est à cause d'une combinaison de plusieurs erreurs.

Le procès qui a eu lieu fut suivit au quotidien par tous les journaux. La décision finale en 1993 signale que sur les 25 patients surexposés, 15 moururent directement par l'irradiation et dans 2 autres cas la sur-irradiation fut un facteur significatif. On condamna la compagnie General Electric, jugée responsable de l'accident.

La société espagnole de physique médicale (SEFM) réalisa un rapport sur l'accident et assura que l'origine des causes était (SEFM, 1991) :

- l'intervention technique incorrecte,

- des déficiences d'organisation,

- le retard dans la détection du dysfonctionnement de l'équipe.

En analysant a posteriori cet accident qui n'a eu en dehors des patients aucune incidence sur la radioprotection de travailleurs et du public, le conseil de sécurité nucléaire (CSN) a estimé qu'il y avait eu des erreurs (CSN, 1991) :

- de communication,

- dans la ligne de responsabilité,

- sur l'accomplissement des procédés.

Cependant, cet accident a mis également en évidence une absence de :

- formation des techniciens de maintenance des entreprises d'électromédecine,

- dispositions légales spécifiques en matière de radiothérapie,

- organisation des unités de radiothérapie et de physique médicale au niveau national,

- ressources humaines et d'équipement dans ces unités,

- formation réglementée du physicien médical.

De plus, cet accident révèle une absence d'entente entre les tutelles responsables de la radioprotection des travailleurs et du public (en Espagne, le CSN est compétant) et celle des patients (la compétence revient au ministère de la Santé) et une responsabilité partagée des physiciens médicaux avec les autres professionnels.

\section{Actions entreprises en Espagne}

Les actions entreprises en Espagne dans les années 90, après avoir tiré les leçons de cet évènement ont été d'adapter la législation aux directives 96/29/Euratom (CCE, 1996) et 97/43/Euratom (CCE, 1997) publiées à partir des recommandations de la publication 60 de la CIPR (ICRP, 1991). Il en a résulté les décisions suivantes :

- création de la spécialité de radiophysique hospitalière (RFH), par le ministère de l'Éducation et de la Science et par le ministère de la Santé (Décret royal, 1997a), 
- établissement par le ministère de la Santé, à partir de 1997, de normes de base pour les critères de qualité en radiothérapie, médecine nucléaire et radiodiagnostic (Décret royal, 1997b, 1998, 1999),

- établissement de programmes de formation en radioprotection par le CSN et le ministère de la santé (Décret royal, 1990, 1992 ; MSC, 2006).

\section{Organisation des services de radiophysique et radioprotection au sein des établissements de santé}

L'organisation des services de radiophysique et radioprotection (SRPR) a été établie, dans un premier temps en 1990, par l'Institut national de la santé (INSALUD) dépendant du ministère de la Santé (MSC, 1990) et de nos jours par les organismes de la santé des gouvernements autonomes. Le CSN, dans le cadre de la radioprotection des travailleurs et du public, a la faculté de décider quels établissements sanitaires doivent disposer de ces services, il les autorise et les contrôle périodiquement.

Le critère du CSN est que tout centre sanitaire disposant d'installations de radiothérapie, médecine nucléaire et radiodiagnostic doit avoir un SRPR à temps plein. Une fois que le CSN l'a exigé, la direction du centre sanitaire doit le mettre en œuvre avec les ressources humaines et techniques nécessaires dans un délai d'un an. Dans le cas contraire, le centre sanitaire sera sanctionné (CSN, 2005).

Ces services ont permis de rassembler, au sein d'une même entité, les radiophysiciens qui étaient auparavant répartis entre les services de radiothérapie, de médecine nucléaire et de radiodiagnostic. Ils sont indépendants des services opérationnels et ils ont un lien hiérarchique direct avec la direction médicale de l'établissement.

Les objectifs et les missions des SRPR sont bien définis dans la réglementation espagnole (Décret royal, 2001b). D'une manière générale, les principaux objectifs du SRPR sont les suivants :

- protéger les patients, les travailleurs et le public en général des risques provenant de l'exposition aux rayonnements ionisants,

- collaborer avec les services de radiothérapie, médicine nucléaire et radiodiagnostic pour suivre les critères de qualité établis par la législation en cette matière,

- réaliser les traitements et les suivis dosimétriques correspondant à la dosimétrie clinique en accord avec la prescription du médecin spécialiste en radiothérapie. L'unité de radiophysique en charge de la dosimétrie clinique doit avoir un lien direct et indépendant avec l'unité médicale de radiothérapie. 
Les activités du SRPR s'articulent aussi autour des thèmes suivants :

- surveillance dosimétrique des travailleurs exposés et des postes de travail,

- projets de construction des installations de radiothérapie, de radiodiagnostic et de médecine nucléaire,

- conseils en radioprotection lors de l'achat de nouveaux équipements,

- acceptation et détermination de l'état de référence initial des équipements, générateurs de rayons $X$ et des systèmes de planification et de calcul de doses, contrôles périodiques associés,

- information et conseils sur l'optimisation de la radioprotection,

- formation en radioprotection des travailleurs, non seulement pour répondre aux exigences réglementaires, mais également pour permettre une vraie sensibilisation à la radioprotection.

La gestion de la radioprotection des centres ne disposant pas de SRPR à temps plein (ayant seulement, par exemple, des installations de médecine nucléaire ou de radiodiagnostic) dépend d'un SRPR d'un grand hôpital ou d'une unité technique de radioprotection (UTPR), unité privée créée pour fournir les services de radioprotection réglementaires. Les UTPR doivent être aussi autorisées et contrôlées par le CSN (CSN, 2005).

Le SRPR, comme tous les autres services hospitaliers, gère ses propres ressources humaines et techniques et assure un rôle de conseiller et de suivi pour les achats des matériel de radioprotection et des équipements de dosimétrie pour tous les services hospitaliers (Badajoz et al., 2009).

\section{Le spécialiste en radiophysique hospitalière}

Entre 1997 et 1999, la fonction de «spécialiste en radiophysique hospitalière » (RFH), équivalent à l' « expert en physique médicale » (CCE, 1997) a été créée et ses missions ont été définies par plusieurs décrets (Décret royal, 1997a, 1998).

Les RFH doivent avoir une formation de 3 ans (la commission nationale de RFH a fait une démarche auprès du ministère de la santé pour le passer à 4 ans) en milieu hospitalier dans le programme général de formation des spécialistes sanitaires (6 mois en radioprotection, 6 mois en médecine nucléaire, 6 mois en radiodiagnostic et 18 mois en radiothérapie). Cette formation est réalisée dans un des 33 SRPR accrédités par le ministère de la Santé avec un programme établi par la commission nationale de la spécialité en RFH. Chacun de ces SRPR a la capacité de former un ou deux RFH par an. L'accès à cette formation se fait par concours national (entre 250 et 300 personnes se présentent pour 33 postes par an). Ils disposent d'un contrat pour 3 ans et perçoivent un salaire mensuel comme le reste de médecins en formation. 
Ces spécialistes sont chargés, à part les missions de radioprotection des travailleurs, du public et des patients, de la mise en œuvre de la dosimétrie, du contrôle qualité des équipements et des installations, de la planification et du calcul des doses délivrées aux patients en radiothérapie. Ils ont un statut équivalent à celui des autres spécialistes médicaux (cardiologues, oncologues, radiologues...).

L'Espagne dispose actuellement d'environ 450 RFH. Malgré l'existence des recommandations de l'European Federation of Organisations for Medical Physics (EFOMP), les effectifs de RFH sont souvent restreints : le nombre de personnes formées à cette fonction est insuffisant. En fait, en Espagne, il y a actuellement une offre de poste de travail qui ne peut pas être satisfaite faute de RFH.

La formation des spécialistes sanitaires en Espagne (système basé sur une formation dans des unités d'enseignements dans des hôpitaux pendant des périodes entre 3 et 5 années selon les spécialités, appelé « médecin interne résident (MIR) ») doit fournir les spécialistes dont a besoin le pays, en tenant compte également des besoins dans le secteur privé (Décret royal, 2008). Une vision limitée, qui pourrait avoir comme objectif de répondre seulement aux besoins des hôpitaux publics, pourrait sûrement provoquer dans le futur un déficit de spécialistes.

Pour évaluer les besoins des RFH à moyen terme, il faut prendre en compte une série de facteurs comme le nombre actuel d'établissements sanitaires disposants d'installations de radiothérapie (selon les données du CSN, il y a 133 installations en 2009) et prévus dans le futur (selon la même source d'information, il est prévu une croissance de 4,5\% annuelle), le rapide développement de la haute technologie diagnostique et thérapeutique (qui exige un plus grand nombre de RFH à cause d'un temps supplémentaire consacré aux nouvelles et complexes techniques), le nombre de spécialistes qui seront en retraite, etc.

Le ministère de la Santé a fait un modèle de simulation pour connaître la situation actuelle et future à moyen et long terme des différentes spécialités sanitaires. Bien qu'il n'y a pas inclus les RFH, on peut estimer, suivant ce modèle, que les besoins de ces spécialistes pour l'année 2015 seront à peu prés de 580 ( $28 \%$ de plus qu'aujourd'hui). Avec le rythme de formation de $33 \mathrm{RFH} / \mathrm{an}$, on estime que les besoins seraient couverts (l'objectif est d'arriver a un compromis raisonnable entre l'offre et la demande) en 4 ans.

Si l'on tient compte que $70 \%$ des RFH travaillent exclusivement en radiothérapie et que le nombre total de médecins radiothérapeutes est de 492 à ce jour (625 sont prévus en 2015) le ratio de médecin oncologue radiothérapeute/RFH est donc de 1,8 et que ce même ratio passerait en 2015 à 1,5. 


\section{Établissement des normes de base sanitaires pour les critères de qualité dans les installations radioactives et radiologiques}

L'Espagne a transcrit dans sa réglementation les recommandations de la CIPR en matière sanitaire (ICRP, 1996), les dispositions contenues dans la directive 97/43/Euratom, sur la protection de la santé face aux risques des radiations ionisantes dues aux expositions médicales (CCE, 1997) selon plusieurs décrets :

- établissement des critères de qualité en médecine nucléaire (Décret royal, 1997b),

- établissement des critères de qualité en radiothérapie (Décret royal, 1998),

- établissement des critères de qualité en radiodiagnostic (Décret royal, 1999),

- justification de l'utilisation des radiations ionisantes dans les expositions médicales (Décret royal, 2001a).

L'objectif de ces critères de qualité est assurer l'optimisation des procédures diagnostiques et thérapeutiques, la radioprotection du patient grâce à l'implantation de programmes d'assurance qualité qui s'appliquent à toutes les unités médicales.

Dans le cas spécifique des installations de radiothérapie, le programme d'assurance qualité envisage toutes les phases du processus radiothérapeutique :

- définition des objectifs,

- description des procédés utilisés, des programmes de contrôle associés, des ressources minimales, en hommes et matériels, nécessaires pour réaliser ces procédés, et des responsables de chaque décision ou procédés, indiquant son niveau d'autorité,

- description des étapes du processus radiothérapeutique et des preuves de contrôle prévues pour ces étapes et pour les dispositifs associés à chacunes d'elles, inclus l'état de référence initiale,

- description du système d'évaluation et analyse des résultats du processus radiothérapeutique.

Ce programme doit être réalisé par les oncologues radiothérapeutes et les RFH. Il doit être approuvé par la commission d'assurance qualité et de contrôle de qualité en radiothérapie formée par un représentent de la direction de l'établissement, le chef du service d'oncologie radiothérapeutique, le chef du service de radiophysique et radioprotection, ainsi que des spécialistes de l'unité médicale et de l'unité de RFH et d'autres personnes à déterminer.

Les autres leçons tirées de l'accident de Saragosse, se concrétisèrent dans ce décret par :

- l'article 15 dans lequel on établie le programme de contrôle de qualité «N'importe quelle anomalie de fonctionnement ou soupçon d'anomalie ou 
n'importe quelle réaction non attendue des patients traités, seront mises immédiatement à la connaissance du médecin responsable de l'unité et du RFH»;

- dans l'article 16 sur le programme de maintenance, «toute réparation ou intervention doit être autorisée par un RFH. Après la réparation de l'équipement réalisée par l'entreprise d'assistance technique, le RFH réalisera les vérifications nécessaires pour vérifier que les niveaux de référence s'accomplissent et, en cas favorable, il remettra l'équipement au médecin responsable de l'unité qui autorise la reprise des traitements». En plus, l'autorisation de l'installation fournie par le CSN stipule que « toute installation de radiothérapie doit avoir un accord formel d'entretien avec entreprises d'assistance techniques autorisées pour les révisions périodiques».

Une annexe du décret précise toutes les preuves, tolérances, périodicité et spécifications pour fixer l'état référentiel initial de l'équipement de radiothérapie et son futur contrôle de qualité. Dans le programme d'assurance qualité figure la réalisation des mesures journalières de photons et électrons, de doses, d'uniformité et d'énergie. Néanmoins, il est nécessaire d'actualiser cette annexe car dans ces 10 dernières années, de nouvelles techniques en téléthérapie et brachythérapie sont apparues.

\section{Établissement de programmes de formations obligatoires en radioprotection}

En accord avec la directive européenne, il existe deux niveaux pour les «PCR » :

- l'expert radioprotection: licencié ou équivalent ; 309 heures de formation supérieure à la radioprotection. Le diplôme est délivré par le CSN. Dans le cas des RFH, le CSN fait passer directement un examen théorique et pratique pour leur délivrer ce diplôme,

- le technicien radioprotection: formation professionnelle ; 40 heures de formation à la radioprotection. Ils travaillent sous les ordres du chef de service de radioprotection.

La réglementation espagnole exige que tout professionnel de santé susceptible d'être exposé soit formé à la radioprotection (patients et travailleurs). Tous les travailleurs exposés avec une "licence requise " (licence de superviseur ou d'opérateur) doivent suivre des cours de formation théorique et pratique, dont le nombre d'heures varie en fonction du domaine (rayons X, médecine nucléaire, radiothérapie, ...) et de la spécialité (médecin ou opérateur). Pour les médecins, cette formation est dispensée lors de l'internat : elle est obligatoire pour obtenir le diplôme et donc pour exercer :

- le médecin radiothérapeute reçoit une formation de 63 heures,

- le médecin nucléaire reçoit une formation de 53 heures, 
- le médecin radiologue reçoit une formation de 25 heures,

- les médecins acteurs de la radiologie interventionnelle (radiologues vasculaires, cardiologues, urologues, gastro-endoscopistes, etc.), en complément de la formation de base de radioprotection en radiologie ( 25 heures) doivent suivre un cours de 20 heures, en accord avec le guide EC RP 116 « Guidelines on Education and Training in Radiation Protection for Medical Exposures $\gg(\mathrm{CCE}$, 2000a).

Pour les médecins n'étant pas conduits à travailler directement avec les rayonnements ionisants (en particulier les médecins prescripteurs) une formation de 10 heures est également requise par le ministère de la Santé. Cette formation est délivrée lors de la période de formation à l'hôpital (MSC, 2006).

Dans la région de Madrid, cette formation a été adaptée aux professionnels concernés, avec un programme pratique qui présente les utilisations des rayonnements en médecine nucléaire, radiologie, radiothérapie et « les bénéfices » que l'on peut en tirer ainsi que quelques notions de physique (les unités), les risques, la radiobiologie, etc. et des notions de radioprotection, pour savoir comment augmenter les bénéfices en diminuant les risques. Il est expliqué également la responsabilité de la justification dans la prescription, spécialement dans les cas de hautes doses, des enfants, des femmes enceintes, selon le guide EC PR 118 (CE, 2000b). Cette formation est très bien acceptée par tous les médecins, selon les opinions obtenues à l'issue de ces cours.

\section{Augmentation des compétences du conseil de sécurité nucléaire}

Depuis 2007, la loi (Loi, 2007) stipule que le CSN, qui n'était jusque-là pas impliqué dans la radioprotection des patients, doit « collaborer avec les autorités compétentes (ministère de la Santé et organismes de santé des autorités régionales autonomes) pour les programmes de radioprotection des personnes soumises à des procédés de diagnostic ou de traitements médicaux utilisant les rayonnements ionisants ».

\section{Conclusions}

La réglementation espagnole en ce qui concerne la radioprotection des patients est mise à jour en tenant compte des derniers avancements technologiques et assure la formation et les besoins des spécialistes en RFH et PCR qui demande le pays. Elle est basée dans le strict respect de nos accords internationaux et elle garantit la sécurité et la radioprotection du patient. 


\section{LEÇONS TIRÉES DE L'ACCIDENT D’UN ACCÉLÉRATEUR LINÉAIRE}

\section{RÉFÉRENCES}

Arranz L. (2008) Gli incidente gravi di radioterapia nel mondo e il loro principali insegnamenti, Convegno Nazionale di Radioprotezione, AIRP, Giugno 2008.

Badajoz C., Bataille C., Drouet F., Schieber C. (2009) Organisation de la Physique Médicale et de la radioprotection : Retour d'expérience de quelques établissements de santé français et étrangers. Rapport n $\mathrm{n}^{\circ}$ 306, CEPN, janvier 2009.

CCE (1984) Euratom, Directive 84/466/Euratom du Conseil du 3 septembre 1984 fixant les mesures fondamentales relatives à la protection radiologique des personnes soumises à des examens et traitements médicaux, JOCE L-265 du 05/10/1984.

CCE (1996) Euratom, Directive 96/29/Euratom du Conseil du 13 mai 1996 fixant les normes de base relatives à la protection sanitaire de la population et des travailleurs contre les dangers résultants des rayonnements ionisants, JOCE L-159 du 29/06/1996.

CCE (1997) Euratom, Directive 97/43/Euratom du Conseil du 30 juin 1997 relative à la protection sanitaire des personnes contre les dangers des rayonnements ionisants lors d'expositions à des fins médicales, remplaçant la directive 84/466/Euratom, JOCE L-180 du 09/07/1997.

CCE (2000a) European Commission, Guidelines on education and training in radiation protection for medical exposures, Radiation Protection 116.EC.2000, European Commission. Guidelines on education and training in radiation protection for medical exposures, Radiation Protection 116.EC.2000.

CCE (2000b) European Commission. Guidelines for healthcare professionals who prescribe imaging investigations involving ionizing radiation, Radiation Protection 118.EC. Update 2003

CSN (1991) Informe sobre el accidente ocurrido en el acelerador lineal del Servicio de Radioterapia del hospital clínico de Zaragoza, en diciembre de 1990, CSN, Marzo 1991.

CSN (2005) Instrucción IS-08 de 27 de julio de 2005 del Consejo de Seguridad Nuclear, sobre criterios exigidos a los titulares de las instalaciones nucleares y radiactivas, el asesoramiento en protección radiológica.

Décret royal (1982) Reglamento de Protección Sanitaria contra Radiaciones Ionizantes, BOE, 8 octubre 1982.

Décret royal (1990) 1132/1990. Protección Radiológica de las personas sometidas a exámenes y tratamientos médicos, BOE, 18 septiembre 1990.

Décret royal (1992) Reglamento de Protección Sanitaria contra Radiaciones Ionizantes, BOE, 12 febrero 1992.

Décret royal (1997a) 220/1997, Creación y regulación de la obtención del título oficial de Especialista en Radiofísica Hospitalaria, BOE, 1 marzo 1997.

Décret royal (1997b) 1841/1997, Establecimiento de los criterios de calidad en medicina nuclear, BOE, 19 diciembre de 1997.

Décret royal (1998) 1566/1998, Establecimiento de los criterios de calidad en Radioterapia, BOE, 28 agosto de 1998.

Décret royal (1999) 1976/1999, Establecimiento de los criterios de calidad en radiodiagnóstico, BOE, 29 de diciembre de 1999.

Décret royal (2001a) 815/2001 sobre justificación del uso de las radiaciones para la protección radiológica de las personas con ocasión de exposiciones médicas. BOE, 14 julio 2001.

Décret royal (2001b) 783/2001, Reglamento de Protección Sanitaria contra Radiaciones Ionizantes. BOE, 26 julio de 2001.

Décret royal (2008) 183/2008. Determinación y clasificación de las especialidades en ciencias de la salud y desarrollo de aspectos del sistema de formación sanitaria especializada, BOE, 21 febrero 2008. 
IAEA (1994) International Basic Safety Standards for Protection against Ionizing Radiation and for Safety of Radiation Sources. Safety Series No 115. Vienna. 1994.

ICRP Publication 26 (1977) Recommendations of the International Commission of Radiological Protection, Ann. ICRP 1 (3).

ICRP Publication 60 (1991) Recommendations of the International Commission of Radiological Protection, Ann. ICRP 21 (1-3).

ICRP Publication 73 (1996) Radiological Protection and Safety in Medicine, Ann. ICRP 26 (2).

Kohn L.T., Corrigan J.M., Donaldson M.S., Eds. (1999) To Err Is Human: Building a Safer System. Washington, DC: National Academy Press, 1999.

Loi (1980) Ley 15/1980 Creación del Consejo de Seguridad Nuclear, BOE, 25 abril 1980.

Loi (1986) Ley 14/1986 Ley General de Sanidad, BOE, 29 abril 1986.

Loi (2007) Ley 33/2007 de reforma de la Ley 15/1980 de creación del Consejo de Seguridad Nuclear. BOE, 8 noviembre 2007.

MSC (1990) Resolución C-7/90 (5-11) del Ministerio de Sanidad y Consumo. Instituto Nacional de la Salud sobre la Creación de las Unidades de Protección Radiológica, 5 noviembre 1990.

MSC (2006) Resolución del Ministerio de Sanidad y Consumo sobre formación en protección radiológica, 21 abril 2006.

SEFM (1991) Informe del accidente ocurrido en el acelerador lineal de electrones del hospital clínico de Zaragoza, entre los días 7 al 20 de diciembre de 1990, SEFM 1/1991, junio 1991. 